\title{
Learning the vocabulary of Russian as a foreign language within the elementary level through Quizlet.com
}

\author{
[Изучение лексики элементарного уровня общего владения \\ русского языка как иностранного при помощи инструмента \\ портала поддержки обучения Quizlet.com]
}

\author{
Kholisakhon Eshmatovna Ismailova - Olga Victorovna Bondareva
}

DOI: 10.18355/XL.2018.11.03.08

\begin{abstract}
The paper presents the methodology and results experimental vocabulary learning Russian as a foreign language by using the support portal training Quizlet.com used with the purpose of increase of efficiency of mastering of a material of an elementary course of the Russian language in the conditions of short-term training to students coming to study in Russia. The article describes the application of the developments of the teacher of Russian as a foreign language in the classroom and independent work of students. The analysis of the obtained results proved the efficiency of the portal tools complex application Quizlet.com for faster development of students' general and special vocabulary, as well as the formation of their basic skills of listening, reading and writing in the studied language.
\end{abstract}

Key words: teaching vocabulary, Russian as a foreign language, electronic means of teaching, independent work of students

\begin{abstract}
Аннотация
В работе представлено описание методики и некоторые результаты применения инструментов портала поддержи обучения Quizlet.com для повышения эффективности ускоренного освоения элементарного курса русского языка как иностранного (РКИ) группой студентов, прибывших на обучение в Россию. Описано применение разработок преподавателя РКИ на занятиях и в режиме самостоятельной работы студентов. Анализ результатов применения флэш-карт, тестов и групповых игр показал перспективность применения всего комплекса инструментов портала Quizlet.com для ускоренного освоения студентами общей и специальной профессиональной лексики РКИ, также формирования у них основных навыков аудирования, чтения и письма на русском языке.
\end{abstract}

Ключевые слова: обучение лексике, русский язык как иностранный, электронные средства обучения, самостоятельная работа студентов

\section{1. Введение}

В связи с развитием мировых интеграционных процессов в сферах экономики, культуры и образования концепция многоязычия студентов и будущих специалистов становится всё более важной и актуальной. Стремление жителей планеты овладеть одним или несколькими иностранными языками, чтобы использовать их для общения и взаимного понимания, проявляется всё активней. Русский язык, как язык международного общения, изучают в разных странах мира. Чтобы изучать русский язык в русскоязычной среде иностранцы приезжают в Россию. Одним из учебных центров, где русский язык как иностранный (РКИ) преподается иностранным гражданам с элементарного уровня, является Факультет русского языка и общеобразовательных дисциплин Российского университета дружбы народов (ФРЯ и ОД РУДН). На факультете, наряду с учебно-методическими пособиями традиционного вида, преподаватели 
разрабатывают и применяют на практике современные инновационные средства поддержки обучения.

Данные разработки основываются на работах теоретического характера, посвящённых методике обучения иностранному языку с использованием новых информационно-коммуникационных интернет технологий (Azimov, 1996; Vlasov, 1990; Kameneva, 2013; Sysoev 2010), в том числе лексике на начальном этапе обучения (Bagaeva, 2006; Fadeev, 1992). Также за основу берутся актуальные исследования по следующим направлениям:

-изучение закономерностей и психологических факторов, влияющих на процесс усвоения русского языка иностранцами (Rosanova, 2017);

-технологии создания лингвометодических тренажеров (Garcov, 2013);

-использование игровых образовательных технологий в системе обучения русскому языку как иностранному (Garcov, 2004; Matohina, 2017).

Принимаются во внимание исследования в области электронной лингводидактики по направлениям:

-исследование возможностей использования информационных технологий в рамках обучения русскому языку (Atabekova, 2008; Bondareva, 2013; Karamysheva, 2000);

-анализ эффективности обучения в условиях модернизации и компьютеризации образования (Beloglazov, 2017).

Авторские электронные информационные продукты преподавателей РКИ, о которых говорилось выше, предназначены для применения в аудиторных и в домашних условиях. На факультете занятия могут проводиться в компьютерных классах. Компьютеры подключены к сети интернет. Широкое распространение мобильных устройств и интернет-технологий позволяет дополнить ими спектр программно-технических средств обучения иностранцев русскому языку. Это также открывает новые перспективы в направлении создания и применения на практике новых информационно-педагогических технологий.

В настоящей работе рассмотрены некоторые элементы информационно-технологической онлайн - поддержки изучения РКИ, использованные нами для повышения эффективности и сокращения сроков прохождения элементарного уровня языковой подготовки студентов. Разработанные для студентов группы информационные продукты преподавателя специально ориентированы на использование инструментов портала Quizlet.com (http:/quizlet.com.ru) в различных режимах - аудиторных, самостоятельных и целевых внеаудиторных мероприятий на базе стационарных компьютерных классов, мобильных компьютерных аудиторий и BYODтехнологий (от англ. Bring Your Own Device) с применением студентами собственных мобильных устройств доступа в интернет (Ismailova, 2016, 2017). В первую очередь - набора электронных флэш-карточек, как основного компонента изучения русских слов, терминов и их определений, а также встроенных систем озвучания и визуальной поддержки на базе внутренней библиотеки изображений Quizlet. B том числе и обучающих групповых игр онлайн-системы Quizlet.live по различным категориям и разделам курса РКИ в аудитории и на внеаудиторных занятиях.

\section{2. Методы}

1. аналитический:

- анализ современных информационных технологий, учебнометодической литературы, практических материалов по обучению РКИ, в частности, лексике;

XLinguae, Volume 11, Issue 3, June 2018, ISSN 1337-8384, eISSN 2453-711X 
2. гипотетико-дедуктивный:

- выдвижение рабочей гипотезы исследования;

3. метод педагогического наблюдения:

- наблюдение за учебным процессом.

3. Цель - анализ возможностей создания элементов ИТ-поддержки процессов формирования и развития у иностранных студентов элементарной языковой коммуникативной компетентности на основе инструментов обучающей онлайн среды Quizlet.com.

\section{4. Задачи}

В задачи нашей работы входило определение и поиск путей решения комплекса проблем:

1. Анализ возможностей pecypca Quizlet.com с точки зрения применения его инструментов для решения задач формирования у иностранных студентов базовых языковых коммуникативных компетенций в рамках элементарного курса РКИ.

2. Формирование у обучающихся элементарных навыков устного общения в образовательно-профессиональной сфере на русском языке как иностранном.

3. Формирование базовых коммуникативных компетенций в соответствии с требованиями стандартов и программ элементарного уровня РКИ (Vladimirova, 2001; Andryushina, 2013).

4. Развитие образовательной ИТ-коммуникативной компетентности будущего студента и специалиста в части применения современных технологий в обучении и самообразовании.

5. Разработка элементов инновационных педагогических технологий с применением инструментов pecypca Quizlet.com на основе стационарного и мобильного доступа в интернет.

\section{5. Методика}

Необходимость поиска путей повышения эффективности обучения, в первую очередь за счет предоставления студентам возможности интенсификации самостоятельной работы над изучением русского языка, обусловлена рядом обстоятельств:

- $\quad$ Время на изучение элементарного курса РКИ значительно сокращено, при этом на изучение грамматики отводится всего 72 часа.

- $\quad$ РКИ воспринимается иностранным учащимся через призму родного языка.

- Языком посредником для студентов является английский язык. Интерфейс программы построен на английском языке. Это позволяет отработать лексический материал с опорой на принцип учёта родного языка учащихся.

- Отсутствует достаточное количество учебно-методической литературы, предназначенной для самостоятельной работы студентов во внеучебное время.

Элементарный уровень - это уровень начальной ориентации в языке, приобретение отдельных знаний и навыков речевой коммуникации. Студент справляется со стандартными фразами простого разговора, использует только выученный материал, его речь состоит из отдельных слов или фраз и понятна носителям языка, привыкшим к общению с иностранцами. На слух студент понимает короткие выученные фразы, требует от говорящего частого повторения и медленного темпа. Он читает знакомые слова и фразы, понимает общее содержание текста, если есть визуальная поддержка и фоновые знания. Может написать выученные слова и фразы, заполнить анкеты и бланки.

При обучении любому иностранному языку необходимо в начале курса хорошо изучать лексику. Именно лексика, взаимодействуя в речи с грамматикой 
и фонетикой, является ведущим средством речевого общения в устной и письменной формах. Лексика неразрывно связана с грамматической системой языка, одно без другого не существует, и лексика оказывает огромное влияние на грамматику. Обучение лексике - это не просто заучивание новых слов, а усвоение имеющихся между ними в языке фонетических, грамматических, смысловых и ассоциативных связей.

Известно, что при изучении русского языка у представителей разных национальностей возникают неодинаковые трудности. Сопоставление структур родного и русского языков облегчает процесс овладения русским языком. Рациональное использование родного языка обучающихся, с одной стороны, способствует эффективному переносу знаний, умений и навыков из сферы родного языка в изучаемый язык. С другой, - помогает преодолеть специфические трудности, обусловленные несоответствием родного и изучаемого языков. В нашем случае тот факт, что родной язык студентов английский, существенно повлиял на выбор программной среды поддержки обучения.

Сайт Quizlet.com - это онлайн-сервис, который служит для того, чтобы помогать быстро запомнить большое количество новых иностранных слов. В том числе - специальных терминов. Идея сервиса проста. Преподаватель создает и добавляет в набор изучаемые слова с переводом (поддерживается большой набор языков). Затем группа приступает к тренировкам. При этом можно добавить изображения, включить автоматическое произношение. Так устанавливается не только визуальный контакт со словом, но и слуховой.

Портал Quizlet.com, как уже говорилось, имеет интерфейс на английском языке, что позволяет исключить дополнительные затраты учебного времени на изучение его устройства. Студенты, для которых английский язык родной, легко ориентируются в этой среде обучения. Одновременно все инструменты данного ресурса допускают применение русского языка для выполнения упражнений и поддержки аудирования.

Создатели портала Quizlet заложили в его конструкцию гибкие возможности. Можно:

- $\quad$ найти и использовать уже готовые сеты с карточками;

- создать свои собственные сеты с необходимой лексикой.

Для проведения занятий и самостоятельной подготовки были разработаны специальные наборы пар слов на английском и русском языке. Пример части одного из наборов показан на рисунке ниже. 


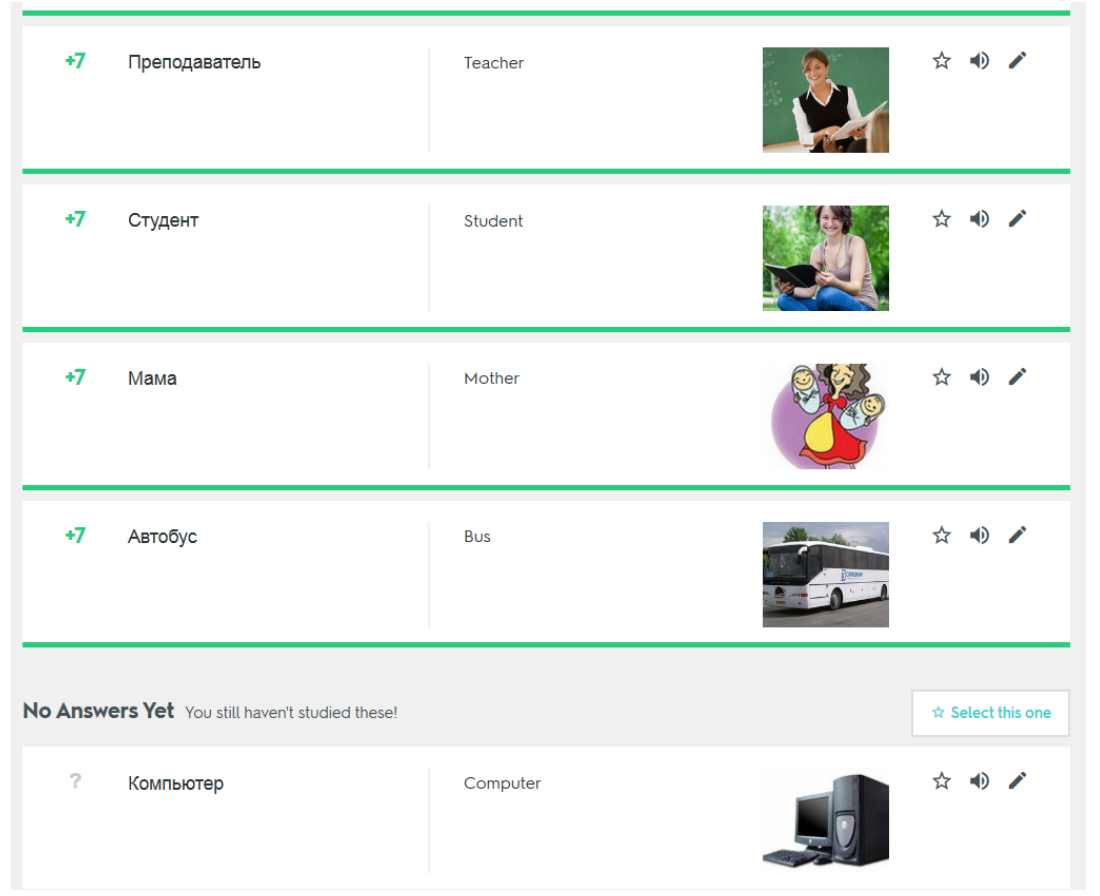

Рисунок 1. Пары русских и английских слов в наборе Quizlet.

Основные инструменты портала включают в себя следующие режимы.

- Режим просмотра карточек

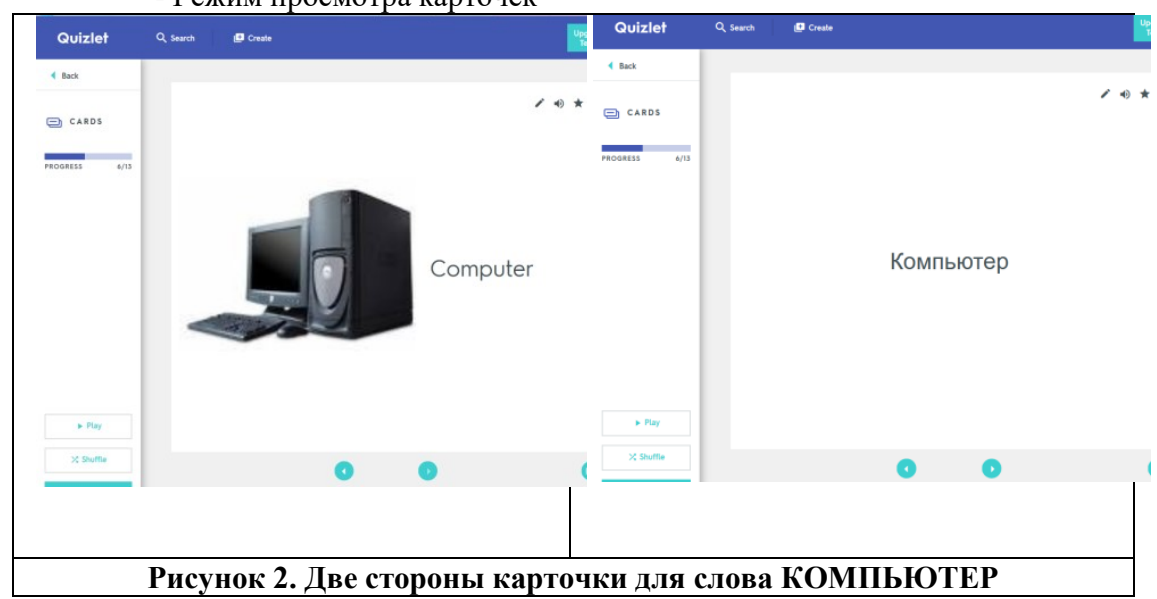

- Режим «LEARN» (введите то, что слышите) 


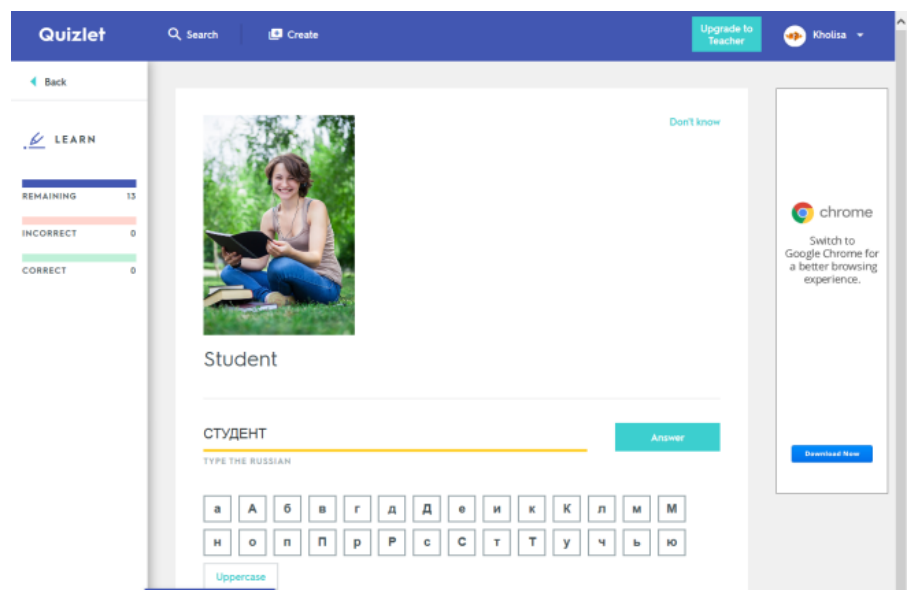

Рисунок 3. Программа LEARN

- Режим «SPELLER» работает в двух вариантах: вписать перевод слова и прослушать значение и записать. Второй способ тренировки наиболее эффективен. Студенту по очереди предлагается 7 слов из сета на родном языке. Ему необходимо самому написать к ним перевод. Все слова из сета нужно написать правильно два раза. Таким образом, если студент ошибется, то данное слово встретится ему еще раз уже в следующем проходе. И так до тех пор, пока он не напишет изучаемую лексему без ошибок.

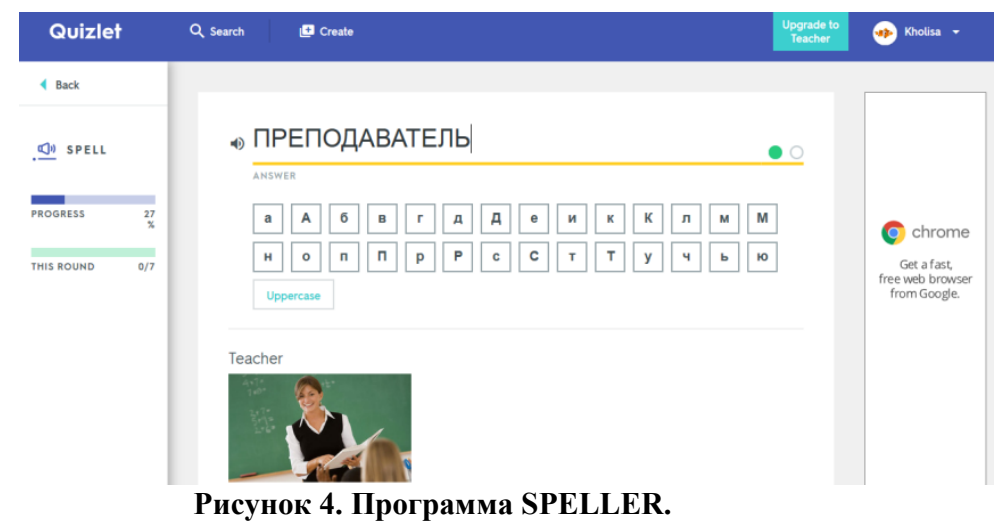

Если студент никак не может вспомнить перевод, можно нажать на кнопку поддержки "Don't know". Перевод отобразится в поле, а затем исчезнет. После этого студенту все равно придется написать данное слово, но, как выученное в этот раз оно не отметится.

После очередного прохода будет доступна краткая статистика результатов учебного сеанса. Студент сможет просмотреть, сколько раз правильно написал слово, и количество оставшихся, еще ни разу не встречавшихся, слов. К переводам можно добавлять и картинки для лучшего восприятия.

- Режим «Тест» автоматически формирует из набора 3 типа вопросов:

- $\quad$ вопрос закрытого типа - вписать перевод слова,

XLinguae, Volume 11, Issue 3, June 2018, ISSN 1337-8384, eISSN 2453-711X 
- $\quad$ вопросы типа «Викторина»,

- $\quad$ вопросы типа « ДА - НЕТ».

По каждому пункту формируется 4 вопроса.

Игра «Match» («Пусть все исчезнет!»). Студент должен при помощи мыши переместить карточку со словом и совместить её с карточкой, содержащей перевод. При правильном совмещении пара карточек исчезает. Компьютер фиксирует время выполнения этого упражнения и ранжирует участников игры по этому параметру. Таким образом, можно организовать очные и заочные соревнование на время.

Игра «Gravity» имеет целью спасение Земли от падающих астероидов. Студент должен ввести правильный перевод слова до того, как астероид упадет на Землю. При наборе определенного количества очков уровень игры повышается и увеличивается скорость падения астероидов. Таким образом, тренируется правильность перевода и отрабатываются навыки скоростного набора текстов на компьютере.

Обучающая среда Quizlet.com содержит очень эффективное средство организации групповой коммуникации в процессе изучения слов. Это встроенная онлайн игра QuizletLive. Как показала практика, использование данного инструмента обеспечивает высокую скорость заучивания правильных вариантов слов.

Использованная нами методика преподавания РКИ предполагает, что студент с первых же занятий начинает «говорить» по-русски. Он не учит отдельные слова - он говорит предложениями, так как модели - это блоки, предложения, сначала примитивные, но постепенно усложняющиеся. И на базе предложения изучаются основные значения падежей и глаголы, управляющие этими падежами. Грамматика усваивается не в результате заучивания падежных окончаний, а в процессе того, что студент постигает значения падежей на тех же моделях. Последовательность введения падежных значений обусловлена частотностью их употребления.

\section{6. Результаты}

Анализируя возможности использования портала Quizlet.com в организации и проведении аудиторных занятий, мы пришли к важному выводу. Внедрение в образовательную практику возможностей данной среды обучения на базе собственных систем мобильного доступа в интернет студентов позволяет нам заметно раздвинуть пространственно-временные рамки образовательной коммуникации всех участников образовательного процесса.

Изучая возможности портала Quizlet.com, мы установили, что эта программа с точки зрения решения задач формирования базовых компетентностей студентов на элементарном уровне РКИ представляет собой продуктивный инструмент семантизации изучаемых русских слов с конкретным значением. Особенно важно то, что одновременное участие аппаратов зрения и слуха способствует более быстрому и прочному усвоению значения нового слова. Перевод в сочетании с демонстрацией изображения и возможностью прослушать звучание русского слова соответствующего объекта - наиболее экономичный и эффективный способ интериоризации значения изучаемого слова.

Описанные выше встроенные инструменты среды Quizlet.com предоставляют широкие возможности для повышения эффективности учебного процесса за счет использования их на занятиях РКИ в компьютерном классе. Ведущую роль здесь играет визуализация слов, понятий, терминов за счет использования библиотеки изображений портала. Демонстрация письменной формы слова на флеш-карточках и его английского аналога сопровождается показом изображения соответствующего объекта. Часто необходимо изучить 
слова, значения которых довольно трудно понять иностранцам, потому что данные предметы или явления отсутствуют в их языковой картине мира. Инструменты pecypca Quizlet.com обеспечивают возможность более полного представления такого рода образа или понятия. Наглядность с поддержкой аудированием способствует развитию у учащихся эмоционально-оценочного отношения к материалу, повышает интерес к знаниям и процессу их получения, позволяет облегчить процесс усвоения лексики элементарного курса РКИ.

\section{7. Заключение}

Результаты проведенных аудиторных занятий и их анализ показали, что применение инструментов портала поддержки обучения Quizlet.com для повышения эффективности ускоренного обучения студентов основам РКИ обеспечивает возможность более продуктивного формирования и развития элементарных навыков речевого общения. В том числе - аудирования, говорения, чтения и письма. Такая форма использования данного онлайнресурса сети интернет открывает широкие возможности для формирования комплекса русскоязычных компетентностей иностранных граждан, проходящих на Факультете русского языка и общеобразовательных дисциплин РУДН предварительную подготовку к обучению в российском университете. Она открывает новые измерения для аудиторной работы и самообразования студентов, помогает направить интеллектуальные способности студентов в освоение РКИ.

Публикация подготовлена при поддержке Программы РУДН «5-100».

The publication was prepared with the support of the "RUDN University Program 5$100 "$

\section{Bibliographic references}

AZIMOV, E. G. 1996. Theory and practice of teaching Russian as a foreign language with the help of computer technology. Thesis for the degree «Doctor of Pedagogical Sciences». Moscow.

ANDRYUSHINA, N.P. - KOZLOVA, T.V. 2013. Lexical minimum in Russian as a foreign language. Elementary level. Common ownership. 5th ed. Saint-Petersburg: Zlatoust. ISBN 978-5-86547-858-4.

ATABEKOVA, A. A. 2008. New computer technologies in teaching Russian as a foreign language: tutorial. Moscow: RUDN.

BAGAEVA, T. V. 2006. Methodical bases of teaching vocabulary with the help of computer technologies (initial stage of training): thesis of a candidate of pedagogical sciences: 13.00.02; [place of conservation: Pushkin State Russian language Institute]. Moscow.

BELOGLAZOV, A. A. - BELOGLAZOVA, L. B. - BONDAREVA, O. V. ISMAILOVA, KH. E. 2017. Monitoring the effectiveness of education in the education modernization and computerization. In: RUDN Journal of Informatization in Education, vol. 14, n. 2, pp. 220-232. ISSN 2312-864X.

BONDAREVA, O. V. 2013. The use of information technologies in the framework of teaching Russian to foreign students. In: Actual problems of modern science, n. 6 (74), pp. 193-195. ISSN 1680-2721.

VLADIMIROVA, T.E. - NAHABINA, M. M. - SOBOLEVA, N.I. ANDRYUSHINA N. P. 2001. State standard for Russian as a foreign language. Elementary level. 2nd edition, revised and enlarged. Saint-Petersburg: Zlatoust. ISBN 5-86547-186-4.

XLinguae, Volume 11, Issue 3, June 2018, ISSN 1337-8384, eISSN 2453-711X 
VLASOV, E. A. - YUDINA, T. F. - AVRAMENKO, O. G. - SHILOV, V. A. 1990. Computers in language learning: problems and solutions. Moscow: Russian language. ISBN 5-200-01058-6.

GARTSOV, A. D. 2004. New information technologies in higher education. Information technologies in language teaching methods: new priorities: a course of lectures. Moscow: RUDN publishing House. ISBN 5-209-02420-2.

GARTSOV, A. D. - GARTSOVA, D. 2013. The Technology of creating linguistic simulators based on the author's workbench. Moscow: RUDN. ISBN 978-5-20905272-2.

ISMAILOVA, KH. E. - MATUKHIN, P. G. - PIVNICKAYA, E. V. 2017. BYOD technologies for a lesson of Russian as foreign language (Maslenitsa Festival for engineering and physics students of preparatory department). In: Russian language abroad, n. 4 (264), pp. 45-56. ISSN 0131-615X.

ISMAILOVA, KH. E. - MATUKHIN, P. G. 2016. Preparing textual elements of byod-technologies in the word-online environment to support elementary skills of Russian speech of foreign students. In: RUDN Journal of Informatization in Education, n. 2, pp. 34-42. ISSN 2312-864X.

KAMENEVA, E. V. 2013. About the role of modern computer technologies in teaching Russian as a foreign language. In: Almanac of modern science and education, n 8 (75), pp. 87-89. ISBN 1993-5552.

KARAMYSHEVA, T. V. Learning foreign languages based on a format of questions and answers using the computer. Saint-Petersburg: Soyuz. ISBN 5-94033-030-4.

MATOKHINA, A.V. - KHARLAMOVA, N. V. - SHABALINA, O. A. KULIKOV, E. A. 2017. The Use of game-based educational technologies in the system of teaching Russian as a foreign language in the modern higher education. In: Open education, vol. 21, n. 3, pp. 39-47. ISSN 2079-5939.

RAKHMANOV, I. V. 1980. Training of oral speech in a foreign language. Moscow: Higher school.

ROZANOVA, S. P. 2017.To the teachers who teach Russian as a foreign language. One hundred and forty-seven useful tips: teaching method manual. 4th ed., erased. Moscow: Flinta: Nauka. ISBN 978-5-9765-2043-1.

SYSOEV, P. V. - EVSTIGNEEV, M.N. 2010. Methods of teaching a foreign language using new information and communication Internet technologies: educational method: tutorial [for students]. Rostov-on-Don, Moscow: Phenix, GlossaPress. ISBN 978-5-222-15997-2.

FADEEV, S. V. 1992. Teaching foreigners Russian vocabulary in order to read texts at the initial stage with the use of computer: thesis of a candidate of pedagogical sciences: 13.00.02; [place of conservation: St. Petersburg State University]. SaintPetersburg.

https:/quizlet.com/ru

Words: 2813

Characters: 22062 (12,26 standard pages)

$\mathrm{PhD}$ in philology, Associate Professor Kholisakhon Eshmatovna Ismailova

Department of Russian language №1

The faculty of Russian language and General educational disciplines

PEOPLES' FRIENDSHIP UNIVERSITY OF RUSSIA (RUDN University)

6, Miklukho-Maklay street

117198, Moscow

Russian Federation

holisa1967@mail.ru

ismailova_khe@rudn.university 
$\mathrm{PhD}$ in pedagogy, Assistant Professor, Olga Victorovna Bondareva

Department of Russian language №2

The faculty of Russian language and General educational disciplines

PEOPLES' FRIENDSHIP UNIVERSITY OF RUSSIA (RUDN University)

6, Miklukho-Maklay street

117198, Moscow

Russian Federation

logiolya@yandex.ru

bondareva_ov@rudn.university 